\title{
Distrofia muscular de Duchenne, presentación clínica
}

\author{
FRANCISCO CAMMARATA-SCALISI ${ }^{1}$, NOLIS CAMACHO ${ }^{2}$, \\ JORGE ALVARADO ${ }^{3}$, MARÍA ANGELINA LACRUZ-RENGEL ${ }^{4}$ \\ 1. Profesor de la Unidad de Genética Médica. Departamento de Puericultura y Pediatría. Universidad de Los Andes. \\ 2. Nutriólogo Pediatra. Profesora del Departamento de Puericultura y Pediatría. Universidad de Los Andes. Instituto \\ Autónomo Hospital Universitario de Los Andes. \\ 3. Pediatra Puericultor. Fundación del Niño Mérida. \\ 4. Pediatra. Neurólogo Infantil. Profesora del Departamento de Puericultura y Pediatría. Universidad de Los Andes. Mérida, \\ Venezuela.
}

\begin{abstract}
Duchenne Muscular Dystrophy clinical presentation

Background: Duchenne Muscular Dystrophy is an X-link recessive disorder that affects 1 per 3.500 males. Becker Muscular Dystrophy is less common, affecting approximately 1 per 30000 males. Both diseases are the result of a mutation in the Xp21 gene that encodes for dystrophin. Objective: Describe the clinical manifestations of Duchenne Muscular Dystrophy in patients at our institution. Method: Observational and descriptive study, in which clinical records of 8 patients with Duchenne Muscular Dystrophy were reviewed, with description of their clinical aspects. Results: The mean age at diagnosis was 5 years-old. 6 boys presented developmental delay and 7 deambulation difficulties, being the main reason for medical attendance. 3 patients died during the study period. Conclusions: A multidisciplinary management is required to delay the disease evolution, while it does not have a curative treatment. It is necessary to know the clinical aspects representative of this disease, in order to perform an early diagnosis.

(Key words: Duchenne Muscular Dystrophy, X-link, recessive, dystrophin).

Rev Chil Pediatr 2008; 79 (5): 495-501
\end{abstract}

\section{RESUMEN}

Introducción: La distrofia muscular de Duchenne es una alteración ligada al X recesiva que afecta 1 en 3500 varones. La distrofia muscular de Becker es menos común, afectando aproximadamente 1 en 30000 varones. Ambas resultan de la mutación de un gen localizado en Xp21, el cual codifica a la distrofina. Objetivos: Describir el comportamiento clínico de la distrofia muscular de Duchenne en pacientes evaluados en nuestra institución. Pacientes y Métodos: Se realizó un estudio de tipo observacional y descriptivo,

Trabajo recibido el 10 de marzo de 2008, devuelto para corregir el 01 de julio de 2008, segunda versión el 21 de julio de 2008, aceptado para publicación el 20 de agosto de 2008.

Correspondencia a:

Dr. Francisco Cammarata-Scalisi

E-mail: francocammarata@yahoo.it 
donde se revisaron las historias clínicas de ocho pacientes con el diagnóstico de distrofia muscular de Duchenne, donde se describieron los aspectos clínicos y paraclínicos de la entidad. Resultados: El promedio de la edad para el momento del diagnóstico fue de cinco años. Seis presentaron retardo del desarrollo psicomotor y la marcha se encontró alterada en siete pacientes siendo este el principal motivo de consulta junto a caídas frecuentes. Tres pacientes habían fallecido al final del período en estudio. Conclusiones: Se requiere de un tratamiento multidisciplinario para retrasar la evolución de la enfermedad, mientras no se disponga de un tratamiento curativo. Es necesario conocer los aspectos representativos de esta enfermedad para realizar su diagnóstico precoz.

(Palabras clave: Distrofia muscular de Duchenne, Ligado al X, recesivo, distrofina).

Rev Chil Pediatr 2008; 79 (5): 495-501

\section{Introducción}

Las distrofias musculares forman parte de una variedad de alteraciones genéticas que se encuentran asociadas a diversas mutaciones de genes que llevan a debilitamiento y atrofia muscular progresiva ${ }^{1}$. La Distrofia Muscular de Duchenne (DMD), es una alteración ligada al $\mathrm{X}$ recesiva, que causa la mutación en el gen distrofina ubicado en $\mathrm{Xp} 21^{2}$. Es fatal y presenta una incidencia estimada en 1 de 3500 nacidos masculinos $^{3}$. El principal hallazgo es el debilitamiento muscular progresivo relacionado con la deficiencia de la proteína sarcolemal de 427$\mathrm{kda}$ denominada distrofina. Existen un amplio rango de síntomas en la enfermedad, que pueden retrasar el diagnóstico ${ }^{4}$, la mayoría de las alteraciones se inician antes de los cuatro años, comúnmente caracterizados por dificultad en la marcha y caídas ${ }^{5}$.

La distrofina es un gen de gran tamaño que consta de 2,6 millones de pares de bases de ADN y contiene 79 exones $^{6}$. En dos tercios de los casos la enfermedad se trasmite por una mujer portadora y el tercio restante proviene de mutaciones de novo, sin historia familiar de la enfermedad. Aproximadamente $60 \%$ de los casos de DMD están asociados con una gran delección intragénica de uno o más exones localizados de manera importante en la región proximal y central del gen (exones 3-7 y 44-55 respectivamente). Alrededor de $6 \%$ de las mutaciones están asociadas con duplicación de un gran segmento y el resto de los casos resulta de mutaciones puntuales, pequeñas delecciones o inserciones ${ }^{7}$. En el siguiente informe haremos referencia de una serie de casos evaluados en nuestra institución para conocer las principales características de presentación clínico-patológicas de la DMD y se realizará una revisión sobre los aspectos clínicos y terapéuticos de este tipo de distrofia muscular.

\section{Pacientes y Métodos}

Se realizó un estudio de tipo observacional y descriptivo, donde se revisaron las historias clínicas de ocho pacientes con el diagnóstico de DMD, en un período de veinte años (19852005), en los Archivos de Historia Médicas del Instituto Autónomo Hospital Universitario de Los Andes. Los datos obtenidos para la realización del estudio se clasificaron usando criterios epidemiológicos: lugar de nacimiento de los pacientes, estratificación social usando el método Graffar Modificado ${ }^{8}$; criterios clínicos: edad de diagnóstico, antecedentes familiares de la enfermedad y personales del paciente, evaluación clínica multidisciplinaria y se empleó la Clasificación Internacional del funcionamiento de la discapacidad y la Salud (CIDDM-2) ${ }^{9}$; paraclínicos: determinación sérica de creatinfosfoquinasa total y de la isoenzima $\mathrm{MB}$, transaminasas, biopsia de músculos gastrocnemio donde se determinó distrofina por inmunotinción y se describió la intervención médica realizada.

\section{Resultados}

Las edades para el momento del diagnóstico presentaron un rango desde los dos años de edad, en un caso, hasta los diez años, en otro; 
con un promedio de 5 años (tabla 1). Cinco de los pacientes eran procedentes del Distrito Sanitario Mérida, donde dos de ellos son hermanos naturales de Mérida, otros dos también hermanos naturales de Colombia y el otro caso es genéticamente independiente. Dos eran naturales del Distrito Sanitario Lagunillas, ubicados en distintas aldeas de los Pueblos del Sur (no consanguíneos), y por último un paciente del Distrito Sanitario Tovar. Según el Método Graffar Modificado, 5 (62,5\%) presentaron estrato social V y $3(37,5 \%)$ IV.

En tres de los pacientes no se reportó antecedentes familiares de la enfermedad y en un caso no pudo ser precisado. En los casos donde se reportaron antecedentes familiares correspondieron a los dos hermanos naturales de Colombia y Mérida, donde todos los pacientes afectados en la familia eran de sexo masculino, lo que suele corresponder a un patrón de herencia ligada al X recesiva. En los antecedentes personales de los pacientes en estudio, seis presentaron retardo del desarrollo psicomotor, cuatro presentaron infecciones en las vías respiratorias, tres fueron asmáticos, los cuales han requerido de múltiples hospitalizaciones y uno presentó politraumatismo no relacionado con la entidad. El paciente que se estudio a partir de los dos años de edad fue valorado inicialmente por retardo del desarrollo psicomotor y referido a la Unidad de Genética Médica por presentar el antecedente de hermano con DMD.

Durante la realización del examen físico de ingreso, la marcha se encontró alterada en siete pacientes donde se describe amplia base de sustentación y basculación, siendo este el principal motivo de consulta junto a caídas frecuentes; dos pacientes no caminaban para el último control. La fuerza muscular braquial y crural se reportó alterada en cinco pacientes, cada uno. La hiporreflexia a nivel braquial se presentó en cuatro y los reflejos rotulianos y aquíleo estaban disminuidos en cinco y cuatro respectivamente. En seis pacientes se reportó la pseudohipetrofia gemelar (figura 1) y en cinco contracturas en el talón. El signo de Gower se reportó positivo en seis pacientes y las escápulas aladas y limitación del codo se presentaron en cuatro pacientes, cada uno (tabla 1).

De los cuatro pacientes con infecciones respiratorias, uno de ellos la presentó de forma recurrente siendo el motivo de consulta dificul-

Tabla 1. Características clínicas en pacientes con DMD

\begin{tabular}{|c|c|c|c|c|c|c|c|c|}
\hline Dato clínico & 1 & 2 & 3 & 4 & 5 & 6 & 7 & 8 \\
\hline Edad de diagnóstico (años) & 5 & 2 & 6 & 3 & 4 & 6 & 4 & 10 \\
\hline Antecedentes familiares & + & + & + & + & - & - & - & NP \\
\hline Retardo psicomotriz & + & + & + & + & - & - & + & + \\
\hline Infecciones respiratorias & $+^{*}$ & + & - & + & - & + & - & - \\
\hline Asmáticos & - & - & + & + & - & - & + & - \\
\hline Alteración en la marcha & + & - & + & + & + & + & + & + \\
\hline Alteración FMB & + & - & + & - & - & + & + & + \\
\hline Alteración FMC & + & - & + & - & - & + & + & + \\
\hline Hiporreflexia braquial & + & - & + & - & - & + & - & + \\
\hline$\downarrow$ de los reflejos rotulianos & + & - & + & - & + & + & - & + \\
\hline$\downarrow$ de los reflejos aquíleo & + & - & + & - & - & + & - & + \\
\hline Pseudohipertrofia gemelar & + & - & + & + & + & + & - & + \\
\hline Contracturas del talón & + & - & + & - & + & + & - & + \\
\hline Signo de Gower & + & NP & + & - & + & + & + & + \\
\hline Escápulas aladas & + & - & - & - & + & + & - & + \\
\hline Limitación del codo & + & - & + & - & + & - & - & + \\
\hline
\end{tabular}

Fuente: Archivos de Historia Médica del I.A.H.U.L.A. NP: No precisado. * Infección en vía aérea recurrente. FMB: Fuerza muscular braquial. FMC: Fuerza muscular crural. 


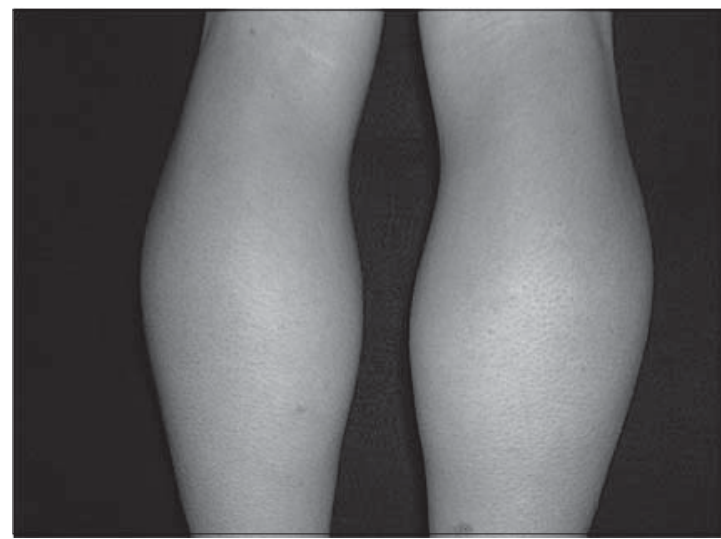

Figura 1. Pseudohipertrofia gemelar en un paciente escolar con diagnóstico de DMD.

tad respiratoria. Entre otros signos clínicos asociados se destacan: cardiopatía y retardo mental en uno y dos pacientes respectivamente. Según la Clasificación Internacional del funcionamiento de la discapacidad y la Salud (CIDDM-2): fue severa en 25\% de los pacientes (imposibilidad para la marcha), 37,5\% moderada (marcha posible con ayuda técnica) y $37,5 \%$ fue leve (ligera dificultad visible, pero no afecta el rendimiento).

El diagnóstico fue corroborado por inmunotinción para distrofina en biopsia de músculo gastrocnemio en todos los pacientes. La toma de muestra se realizó en quirófano por el Servicio de Cirugía Pediátrica. Todos los estudios fueron realizados a partir 1998, sólo en dos pacientes los estudios se realizaron en muestras de músculo anteriormente tomadas y preservadas bloques de parafina, donde posteriormente se le hizo estudio inmunohistoquímico por el Departamento de Anatomía Patológica de la Universidad de Los Andes.

El estudio de creatinfosfoquinasa total en seis pacientes reportó en promedio 67 veces por encima de su rango superior y de la isoenzima MB 23 veces mayor. Las transaminasas igualmente se presentaron elevadas en igual número de pacientes donde la ALT se presentó en promedio diez veces superior a su rango y la AST cinco veces superior. Los dos pacientes en que no se reportó pruebas de laboratorio, tenían el antecedente de un hermano con clíni- ca similar, el cual, había sido estudiado de forma completa, sin embargo, como se expresó anteriormente el estudio inmunohistoquímico se realizó a la totalidad de pacientes. A pesar que estaba indicado realizar pruebas de laboratorios similares a las madres, sólo se contó con reportes de tres de ellas, donde en dos existían valores alterados de creatinfosquinasa, una de ellas es la madre de dos los niños naturales de Mérida, dato bioquímico que puede corroborar su estado de portadora. Sin embargo, estos deben estudiarse más ampliamente.

El tratamiento terapéutico con corticoesteroides se realizó inicialmente en la totalidad de pacientes, mientras estos se podían tener supervisión médica, sin embargo, los dos pacientes procedentes de las diferentes aldeas de los Pueblos del Sur, su seguimiento fue menor de tres controles, debido a lo lejano entre del domicilio y el centro asistencial, así como de los escasos recursos económicos de las familias expresado por el Método Graffar Modificado. Igualmente el tratamiento fisiátrico ha sido realizado en seis pacientes, por lo anteriormente comentado, no obstante, se le indicó a padres y familiares que terapias son recomendables realizar en casa. Para finales del 2005, tres pacientes habían sido reportados como fallecidos, correspondientes a los casos clínicos 3,6 y 8 con edades de 17, 21 y 14 años respectivamente, presentando importante discapacidad motora y fallo cardiorrespiratorio.

\section{Discusión}

La DMD es una enfermedad letal ligada al $\mathrm{X}$ recesiva, por lo que el riesgo de recurrencia en una mujer portadora de la enfermedad en cada gestación es: 50\% de hijos enfermos, 50\% de hijos sanos; $50 \%$ de hijas portadoras y finalmente $50 \%$ de hijas no portadoras. El diagnóstico en una familia crea por consiguiente, la necesidad de detectar a las mujeres portadoras con la finalidad de poder establecer el adecuado asesoramiento genético ${ }^{10,11}$. En la serie estudiada sólo en una familia se pudo realizar el estudio para establecer el estado portador de la madre, el cual fue realizado en un centro de referencia nacional. Los avances en genética 
molecular han ampliado los conocimientos con relación a las distrofias musculares. La clarificación de la patogenia, debida a déficit de una proteína especifica que altera el complejo de proteínas asociadas a la distrofina, ha generado una nueva clasificación basada en el defecto proteico y genómico ${ }^{12}$. Las distrofinopatías ligadas al X, resultan de la mutación en el gen distrofina, que es la causa más común de miopatía heredada en hombres y muestra varios grados de severidad, desde elevación asintomática de la creatinfosfoquinasa, en la Distrofia Muscular de Becker (DMB), que es la forma de presentación leve, hasta la forma severa la DMD cuya enfermedad progresa rápidamente $\mathrm{y}$ puede perder la habilidad de caminar antes de los 12 años. El curso de la DMB es por lo tanto, más benigno y el promedio de edad de inicio de los síntomas como: debilidad muscular y alteración en la marcha es reportado alrededor de los 12 años de edad. El fenotipo clínico y los resultados paraclínicos son relativamente homogéneos en la DMD, como lo expresado en los casos estudiados en este reporte, que no presenta diferencias significativas en la evolución clínica de otras serie de casos presentadas en la literatura, pero que el pediatra debe conocer para diagnosticar y emplear las diversas pruebas complementarias y disponibles para establecer el diagnóstico y brindar una oportuna atención médica al paciente y la familia. A diferencia de la DMB que muestra un perfil más heterogéneo, con poca correlación entre los hallazgos clínicos y de laboratorio ${ }^{13}$.

Los pacientes con DMD pueden presentar un promedio cognitivo inferior y puede ser manifiesto más específicamente en déficit del lenguaje. En 25 pacientes holandeses con un promedio de edad de 10,1 años, cinco presentaron alteraciones graves y otros cinco moderadas de lectura. Estos problemas de lectura eran independientes del nivel de información procesado y el funcionamiento conductual ${ }^{14}$. Análisis de informes en padres en los que se aseguraba la adquisición de precursores tempranos del lenguaje en 130 niños con DMD y 59 hermanos de estos que no estaban afectados. Los niños con DMD fueron más propensos a mostrar retrasos tanto del desarrollo motor como del lengua- je, además tenían bajas puntuaciones cognitivas, en comparación con sus hermanos sanos. Estos datos muestran la necesidad de una intervención temprana, ya que un inicio precoz de ayuda limitará problemas de aprendizaje y esto beneficiará a su calidad de de vida ${ }^{15}$. Un estudio que analiza la percepción de la calidad de vida de los pacientes con DMD realizado en Brasil, reporta que el motivo que más produce infelicidad es el aislamiento social, resultado de la inmovilidad que sufren estos y el segundo motivo es el de las limitaciones impuestas por la enfermedad, aunque en este caso está más presente en la percepción del cuidador que la del propio niño ${ }^{16}$.

A nivel cardiopulmonar la enfermedad causa debilitamiento progresivo de los músculos respiratorios, así como de otros músculos esqueléticos en su curso. La capacidad vital incrementa concomitantemente con el crecimiento físico hasta la adolescencia, sin embargo, comienza a decrecer de forma temprana en pacientes con DMD. La capacidad vital entra en fase de descenso, disminuyendo un promedio de $8,5 \%$ por año, después de los 10-12 años. Como resultado los pacientes desarrollan hipoventilación crónica alveolar en estado avanzado $^{17}$. Las enfermedades cardíacas se manifiestan como cardiomiopatía dilatada y/o arritmias cardíacas ${ }^{18}$. Ikeniwa y col ${ }^{19}$, reportaron dos casos de pacientes de 21 años de edad con DMD complicados con cardiomiopatía dilatada e infarto cerebral, presentado disartria y debilitamiento facial derecho en el primer paciente y disartria acompañada con hemiplejia derecha en el segundo. La muerte principalmente ocurre a partir de los veinte años por falla cardiorrespiratoria, donde los pacientes están usualmente sometidos a ventilación mecánica.

Los avances en las estrategias para el tratamiento de la enfermedad descritos por Nowak y Davies ${ }^{6}$ son: Terapia de células madres, trasplante de mioblastos, terapia de reemplazo del gen, terapia sobrereguladora, inhibidores del proteosoma y corrección precisa de la mutación, entre otras herramientas que se encuentran en fase de experimentación y con resultados diversos. Sin embargo, lo disponible para el manejo hasta el momento en nuestra área son 
medidas médicas, quirúrgicas y de rehabilitación, para optimizar y mantener las funciones. Entre los diferentes medicamentos que han sido probados como tratamientos sólo los corticoesteroides han provisto un mejoramiento temporal. Los resultados muestran un porcentaje bajo de progresión o estabilización de la fuerza muscular; pero los efectos adversos del uso de esteroides crónico no ha permitido el consenso de este medicamento en el tratamiento estándar de la $\mathrm{DMD}^{20}$. King y col${ }^{21}$, estudiaron los efectos ortopédicos del tratamiento a largo plazo con corticoesteroides en 143 jóvenes afectados, el cual el tratamiento con esteroides redujo significativamente la escoliosis durante más de tres años independiente de la ambulación, sin embargo, existe un incremento en el riesgo de fracturas vertebrales y en miembros inferiores en comparación con los pacientes no tratados. El mecanismo de acción de los corticoesteroides es aún incierto, aunque se han propuesto diversas posibilidades basadas principalmente en la observación en el modelo animal y en un número limitado de pacientes en estudio, donde se incluyen:

1. Alteración en los niveles de ARNm estructural, señalización y genes en la respuesta inmune.

2. Reducción de los linfocitos T citotóxicos.

3. Disminución de la concentración y flujo de calcio.

4. Incremento en la expresión de laminina y reparación miogénica.

5. Retardo en la apoptosis muscular e infiltración celular.

6. Realza la expresión de distrofina.

7. Afectación de la transmisión neuromuscular.

8. Atenúa la necrosis de la fibra muscular.

9. Disminuye el porcentaje de daño de la fibra muscular.

10. Aumenta los niveles de taurina y creatina muscular.

No obstante, otros estudios son necesarios para establecer los mecanismos celulares, que demuestren la utilidad del fármaco en la $\mathrm{DMD}^{20}$. Por otra parte se ha reportado que la administración oral de glutamina o suplementación de aminoácidos por más de diez días inhibe la degradación de las proteínas corporales ${ }^{22}$.

Mientras no exista un tratamiento curativo, sólo se puede retrasar la evolución de la enfermedad por medio de un tratamiento multidisciplinario el cual comprende la atención y evaluación: neurológica, nutricional, cardiovascular, respiratoria, ortopédica, psiquiátrica y genética esta última para impartir el oportuno consejo a los padres y familiares de los pacientes. Además es necesario que este tratamiento se desarrolle de manera precoz, regular y permanente para que de esta manera se puedan disminuir los efectos presentes y mejorar la calidad de vida de los pacientes.

Es necesario conocer los aspectos representativos de esta enfermedad para que sea diagnosticada precozmente y a su vez se debe tener en cuenta que existen otros tipos de distrofias musculares, cuyo datos son necesarios para establecer diagnóstico diferencial. Además la realización de una genealogía puede ayudar a orientar el diagnóstico o incluso corroborarlo. Es indispensable realizar un detallado y adecuado examen clínico buscando los síntomas y signos propios de la enfermedad, realizar las pruebas de laboratorio pertinentes comentados en los resultados de este informe, así como la toma de biopsia muscular para determinar el defecto por medio de la realización del estudio inmunohistoquímico y el estudio a nivel molecular de la alteración génica; todo en conjunto para determinar el diagnóstico tanto del enfermo como de la madre y de mujeres portadoras en la familia. En nuestra institución no se realiza el diagnóstico molecular de la alteración como se menciono anteriormente, información importarte para poder correlacionar fenotipo-genotipo. Sin embargo, ante un paciente con sospecha clínica de distrofia muscular, el y su grupo familiar entran en un protocolo de evaluación clínica multidisciplinaria para brindar una atención médica integral. La revisión de casos evaluados como los presentados en este apartado nos aporta datos de interés sobre la evolución clínica de los pacientes, además compromete el grupo de médicos tratantes actualizarse continuamente en los avances científicos referentes a este tipo de alteración letal, para brindar nuevas herramientas en el tratamientos que puedan estar disponibles. 


\section{Referencias}

1.- Tsao CY, Mendell JR: Coexisting muscular dystrophies and epilepsy in children. J Child Neurol 2006; 21: 148-50.

2.- Tay SK, Khng HH, Low PS, Lai PS: Diagnostic strategy for the detection of dystrophin gene mutations in asian patients and carriers using immortalized cell lines. J Child Neurol 2006; 21: 150-5.

3.- Strober JB: Therapeutics in duchenne muscular dystrophy. Neurorx 2006; 3: 225-34.

4.- Parsons EP, Clarke AJ, Bradley DM: Developmental progress in duchenne muscular dystrophy: lessons for earlier detection. Eur J Paediatr Neurol 2004; 8: 14553.

5.- Pereira CC, Kiyomoto BH, Cardoso R, Oliveira AS: Duchenne muscular dystrophy: alpha-dystroglycan immunoexpression in skeletal muscle and cognitive performance. Arq Neuropsiquiatr 2005; 63: 984-9.

6.- Nowak KJ, Davies KE: Duchenne muscular dystrophy and dystrophin: pathogenesis and opportunities for treatment. Embo Rep 2004; 5: 872-6.

7.- Hung CC, Su YN, Lin CY, et al: Denaturing hplc coupled with multiplex pcr for rapid detection of large deletions in duchenne muscular dystrophy carriers. Clin Chem 2005; 51: 1252-6.

8.- Méndez-Castellano H: Estratificación social y biología humana. Arch Venez Puer Ped 1986; 48: 93-102.

9.- World health organization. International classification of functioning, disability and health (icf). Ginebra: 2001.

10.- Delgado-Luengo WN, Borjas-Fuentes L, ZabalaFernández $W$, et al: Detección de portadoras de distrofia muscular de Duchenne/becker a través del análisis de loci STRs ligados al gen de la distrofina en familias venezolanas. Invest Clin 2002; 43: 239-54.

11.- Fonseca D, Tamar C, Mateus H: Detección de portadoras de distrofia muscular de duchenne en familias colombianas mediante análisis de microsatélites. Colomb Med 2008; 39 (supl 2): 7-13.

12.- Erazo-Torricelli R: Actualización en distrofias muscu- lares. Rev Neurol 2004; 39: 860-71.

13.- Ramelli GP, Joncourt F, Luetschg J, Weis J, Tolnay M, Burgunder JM: Becker muscular dystrophy with marked divergence between clinical and molecular genetic findings: case series. Swiss Med Wkly 2006; 136: 18993.

14.- Hendriksen JG, Vles JS: Are males with duchenne muscular dystrophy at risk for reading disabilities? Pediatr Neurol 2006; 34: 296-300.

15.- Cyrulnik SE, Fee RJ, De Vivo DC, Goldstein E, Hinton $V J$ : Delayed developmental language milestones in children with duchenne's muscular dystrophy. J Pediatr 2007; 150: 474-8.

16.- Longo-Araújo de Melo E, Moreno-Valdés MT: Buena calidad de vida de los pacientes con distrofia muscular de duchenne. Rev Neurol 2007; 45: 81-7.

17.- Kang SW, Kang YS, Sohn HS, Park JH, Moon JH: Respiratory muscle strength and cough capacity in patients with duchenne muscular dystrophy. Yonsei Med J 2006; 47: 184-90.

18.- American academy of pediatrics section on cardiology and cardiac surgery. Cardiovascular health supervision for individuals affected by Duchenne or Becker muscular dystrophy. Pediatrics 2005; 116: 1569-73.

19.- Ikeniwa C, Sakai M, Kimura S, et al: Two cases of duchenne muscular dystrophy complicated with dilated cardiomyopathy and cerebral infarction. No to Shinkei 2006; 58: 250-5.

20.- Moxley RT, Ashwal S, Pandya S, et al: Practice parameter: corticosteroid treatment of duchenne dystrophy. Neurology 2005; 64: 13-20.

21.- King WM, Ruttencutter R, Nagaraja HN, Matkovic V, landoll J, hoyle c, et al: Orthopedic outcomes of longterm daily corticosteroid treatment in duchenne muscular dystrophy. Neurology 2007; 68: 1607-13.

22.- Mok E, Violante CE, Daubrosse C, Gottrand F, Rigal O, Fontan JE, et al: Oral glutamine and amino acid supplementation inhibit whole-body protein degradation in children with duchenne muscular dystrophy. Am J Clin Nutr 2006; 83: 823-8. 